Check for updates

Cite this: RSC Adv., 2017, 7, 39452

\title{
Electrospun PCL-PIBMD/SF blend scaffolds with plasmid complexes for endothelial cell proliferation
}

\author{
Lingchuang Bai, (DD ab Qian Li, ${ }^{\text {ab }}$ Xinghong Duo, ${ }^{\text {ab }}$ Xuefang Hao, ${ }^{\text {ab }}$ Wencheng Zhang, ${ }^{c}$ \\ Changcan Shi, (D)*de Jintang Guo, ${ }^{\text {ab }}$ Xiangkui Ren (D)*ab and Yakai Feng*abefg
}

Tissue engineering scaffolds with gene delivery function play an important role in DNA-based vascular tissue engineering. In the present work, we used biodegradable polyester-polydepsipeptide, silk fibroin (SF) and gene complexes to prepare electrospun scaffolds encapsulating gene complexes in order to enhance the proliferation of endothelial cells. A series of nanofibrous scaffolds with different properties including fiber diameter, hydrophilicity, porosity and mechanical properties were prepared by electrospinning technology with adjusting the weight ratio of poly( $\varepsilon$-caprolactone)-b-poly(isobutylmorpholine-2,5-dione) (PCL-PIBMD) and SF. PCL-PIBMD/SF blend scaffolds were optimized to obtain the scaffolds with a weight ratio of $90 / 10$ to have superior mechanical performance and good biocompatibility. pEGFP-ZNF580 plasmid (pZNF580) complexes were electrosprayed onto these PCL$\mathrm{PIBMD} / \mathrm{SF}$ blend scaffolds to promote the proliferation of endothelial cells. In order to maintain the stability and integrity of plasmid complexes loaded in scaffolds, the composite scaffolds were fabricated by alternatively layer-by-layer electrospinning and electrospraying techniques. These composite scaffolds showed obviously low platelet adhesion and good histocompatibility. They could effectively enhance the adhesion, spreading and proliferation of human umbilical vein endothelial cells. These results indicated that the composite scaffolds could serve as an attractive platform to deliver therapeutic genes for vascular tissue engineering.

Received 5th June 2017

Accepted 4th August 2017

DOI: $10.1039 / c 7 r a 06253 b$

rsc.li/rsc-advances

\section{Introduction}

Tissue-engineered vascular grafts have been rigorously pursued as blood vessel substitutes due to the limitation of allografts in clinical utility. ${ }^{1}$ Ideal vascular grafts should not only possess sufficient mechanical strength, good biocompatibility and biodegradability, but also have bioactivity and anticoagulant properties. $^{2}$ However, compared to natural blood vessel, tissueengineered vascular grafts usually encounter the problems of thrombosis and low long-term patency after transplantation due to the lack of complete endothelial cell (EC) layer. ${ }^{3}$ ECs form

${ }^{a}$ School of Chemical Engineering and Technology, Tianjin University, Yaguan Road 135, Tianjin 300350, China. E-mail: yakaifeng@tju.edu.cn; Fax: +86-22-27401999; Tel: +86-22-27401999

${ }^{b}$ Collaborative Innovation Center of Chemical Science and Chemical Engineering (Tianjin), Weijin Road 92, Tianjin 300072, China

'Department of Physiology and Pathophysiology, Logistics University of Chinese People's Armed Police Force, Tianjin 300162, China

${ }^{d}$ Institute of Biomaterials and Engineering, Wenzhou Medical University, Wenzhou, Zhejiang 325011, China

${ }^{e}$ Wenzhou Institute of Biomaterials and Engineering, CNITECH, CAS, Wenzhou, Zhejiang 325011, China

${ }^{f}$ Tianjin University - Helmholtz-Zentrum Geesthacht, Joint Laboratory for Biomaterials and Regenerative Medicine, Tianjin, 300072, China

${ }^{g}$ Key Laboratory of Systems Bioengineering (Ministry of Education), Tianjin University, Weijin Road 92, Tianjin 300072, China the innermost layer of natural blood vessel in vivo and play a vital role in keeping the patency of natural blood vessel. Hence, rapid endothelialization has been extensively investigated with the purpose of improving biocompatibility, preventing intimal hyperplasia and subsequently maintaining the long-term patency of artificial vascular grafts. ${ }^{4-6}$ In order to realize the goal of rapid endothelialization, scaffolds with bioactive molecules, growth factors and gene delivery as well as surface modification have been extensively studied., ${ }^{3-9}$ However, the bioactivity and function of bioactive molecules, growth factors and genes can be easily affected by organic solvents and harsh conditions during the preparation of scaffolds. ${ }^{9,10}$ It is a key point to maintain the complete structure and functionality of cargo in scaffolds so as to keep gene's biofunctions.

Currently, gene therapy presents a new strategy to enhance endothelialization in vascular tissue engineering because a newly formed endothelial layer can rapidly spread on the surface of artificial vascular grafts via transfected ECs. ${ }^{\mathbf{1 1 - 1 4}}$ Compared with growth factor delivery, gene delivery is more efficient and effective, which makes it promising for vascular tissue engineering. Recently, our group developed several kinds of gene complexes with excellent transfection efficiency in vitro. ${ }^{15-21}$ The biodegradable gene carriers were functionalized with targeting REDV peptide to exhibit low cytotoxicity, 
favorable targeting function as well as high transfection efficiency. ${ }^{22}$ This investigation provides an insight that pZNF580 gene complexes can be combined with vascular grafts to promote endothelialization for revascularization. The combination of gene therapy and vascular tissue engineering can result in a powerful synergism of treatment options for regenerative medicine.

DNA-based therapeutics were administrated systematically in vitro and in vivo to stimulate tissue regeneration. ${ }^{23-25}$ However, efficient approaches for delivering plasmid complexes with maintaining their complete structure and functionality to specific tissue via scaffolds were rarely studied. Recently, our group reported that PLGA/SF scaffold and PCL-PIBMD scaffold with plasmid complexes could promote the proliferation of ECs in vitro. ${ }^{26,27}$ However, in view of the bloodstream flowing force in vivo, the stability of plasmid complexes which just attached onto the surface of scaffold is still in doubt. Based on our previous work, we developed a simple and feasible method, named "alternatively layer-by-layer electrospinning and electrospraying techniques", in order to enhance the amount and stability of plasmid complexes loaded in scaffolds. This method could favorably protect plasmid complexes from organic solvents during electrospinning and effectively promote stability of gene complexes simultaneously, which is beneficial to maintain their complete structure and functionality. Luu et al. incorporated plasmid DNA into polymer scaffold using blend electrospinning and indicated that the plasmid DNA released from the scaffold was still capable of cellular transfection. ${ }^{28}$ Although blend electrospinning is considered relatively easy to perform, it may lead to the activity loss of incorporated biomolecules, especially for proteins. ${ }^{9}$ Therefore, it could be assumed that plasmid complexes embedded in a sandwich-like composite scaffold using alternatively layer-bylayer electrospinning and electrospraying techniques could take significant advantages for keeping its own integrity and bioactivity as well as promoting endothelialization.

Electrospun polymeric nanofibers could closely mimic the natural extracellular matrix (ECM) morphology and are attractive materials for localized delivery of therapeutics. ${ }^{29-31}$ Electrospinning technology, which can fabricate nanofibrous scaffolds with high surface area and 3D porous microstructure similar to ECM, is regarded as a simple and efficient method to prepare scaffolds. ${ }^{32,33}$ It could produce nanofibrous scaffolds with fiber diameters varying from nanoscale to several microns, and unique three-dimensional structure for cell proliferation and infiltration as well as nutrient transport. More importantly, it is convenient to encapsulate and deliver biologically active molecules, such as growth factors, ${ }^{34,35}$ siRNAs $^{36-38}$ and DNAs ${ }^{39,40}$ within the electrospun nanofibers to modulate cellular functions and tissue responses. Raftery et al. reported that chitosanpDNA nanoparticles incorporated in collagen-based scaffolds showed high transfection efficiency in mesenchymal stem cells and had potential for therapeutic applications in bone and cartilage tissue engineering. ${ }^{\mathbf{4 1}}$ Zhao et al. delivered rhBMP-2 plasmid DNA complexes to the pre-osteoblastic cell line via a PLLA/collagen electrospun scaffold and induced ectopic bone formation. ${ }^{42}$ However, few reports about scaffold-based plasmid
DNA delivery as well as maintaining the complete structure and functionality of plasmid complexes within composite scaffolds in vascular tissue engineering have been published.

In this paper, on the one hand, we expect to effectively deliver plasmid complexes via composite scaffolds to ECs so as to achieve the combination of gene therapy and tissue engineering in regeneration medicine; on the other hand, we attempt to maintain the complete structure and functionality of plasmid complexes in the scaffolds with the purpose of keeping the bioactivity of scaffolds via a relatively simple and effective electrospinning technology. We have previously demonstrated that REDV peptide-modified pEGFP-ZNF580 plasmid complexes could target-deliver pZNF580 gene to ECs and exhibit high transfection efficiency. ${ }^{22}$ Moreover, REDV peptide has been widely used in surface modification and gene delivery system to selectively target ECs via cell surface receptor integrins. The aim of this paper is to prepare a sandwich-like composite scaffold embedded with plasmid complexes by alternatively layer-bylayer electrospinning and electrospraying techniques using PCL-PIBMD, silk fibroin (SF) and plasmid complexes. It is the first time to prepare PCL-PIBMD and SF blend scaffold, in which PCL-PIBMD provides it with high mechanical strength and SF with cell affinity sites. For the sandwich-like composite scaffold, the layer of plasmid complexes between the nanofiber layers could help better maintain their own integrity and functions, and further regulate the cellular behavior of ECs. Herein, micromorphology, mechanical properties, surface wettability, porosity were evaluated so as to determine the optimized blend PCL-PIBMD/SF scaffolds. Further, the effect of plasmid complexes on nanofiber morphology, hemocompatibility and histocompatibility was evaluated. The composite scaffolds loaded with plasmid complexes were evaluated by cell proliferation, cell spreading and fluorescein diacetate (FDA) staining assay. This strategy is relatively convenient and effective and can be used to create a platform for scaffold-mediated gene therapy in vascular tissue engineering.

\section{Experimental section}

\subsection{Materials}

Poly( $\varepsilon$-caprolactone)- $b$-poly(isobutyl-morpholine-2,5-dione) (PCL-PIBMD) composed of polyester segments and polydepsipeptide segments were synthesized from PCL-diol and PIBMD-diol with 2,2,4-, 2,4,4-trimethylhexamethylene diisocyanate (TMDI) as a coupling agent in previous work. ${ }^{43} \mathrm{SF}$ was extracted from Bombyx mori cocoons as previously described. ${ }^{\mathbf{4 4}}$ 1,1,1,3,3,3-Hexafluoro-2-propanol (HFIP, 99.5\%) was bought from Beijing HWRK Chem Co., Ltd., China. Lactide (LA) and glycolide (GA) were obtained from Foryou Medical Device Co., Ltd. (Huizhou, China). Poly(ethylene glycol) monomethyl ether (mPEG, $\left.M_{\mathrm{w}}=1.9 \mathrm{kDa}\right)$, stannous octoate $\left(\mathrm{Sn}(\mathrm{Oct})_{2}\right)$, branched PEI $\left(M_{\mathrm{w}}=10 \mathrm{kDa}\right)$ and fluorescein diacetate (FDA) were all purchased from Sigma-Aldrich (St. Louis, MO, USA). Dimethyl sulfoxide (DMSO) was purchased from Sigma (St. Louis, MO). CREDVW peptide was obtained from GL Biochem (Shanghai, China) Ltd. pEGFP-ZNF580 plasmids (pZNF580) were preserved by department of physiology and pathophysiology, Logistics 
University of Chinese People's Armed Police Force. Other chemicals were of analytical grade from Jiangtian Chemicals (Tianjin, China).

\subsection{Preparation of spinning solution}

PCL-PIBMD and SF was dissolved in HFIP to prepare $10 \%(\mathrm{w} / \mathrm{v})$ mixed solution with a serious of PCL-PIBMD/SF weight ratios (i.e., 100/0, 90/10, 70/30, 50/50, 30/70, 10/90, w/w). The mixture was agitated overnight on a magnetic stirrer at room temperature to form homogenous solutions.

\subsection{Preparation of MPs and MPs/pZNF580 complexes suspension}

The microparticles (MPs) and MPs/pZNF580 suspension were prepared as our previous work described..$^{22}$ Briefly, mPEG- $b$ PLGA diblock copolymers were synthesized by ring-opening polymerization (ROP) of LA and GA monomers. Then, PEI and CREDVW peptides were conjugated to mPEG- $b$-PLGA diblock copolymers sequentially. MPs composed of MPEG- $b$-PLGA- $g$ PEI-REDV were created via nanoprecipitation technology and MPs/pZNF580 complexes were obtained by adding pEGFPZNF580 (pZNF580) plasmid solution to MPs suspension at N/P molar ratio of 20 , which exhibited high transfection efficiency in endothelial cells. ${ }^{22}$ Transmission electron microscope (TEM, JEM-2100F, Japan) was used to characterize the morphology of MPs and MPs/pZNF580 complexes at $200 \mathrm{kV}$ accelerating voltage.

\subsection{Electrospinning PCL-PIBMD/SF to fabricate nanofibrous scaffolds}

PCL-PIBMD/SF mixed solution with different weight ratio was fed into a $5 \mathrm{~mL}$ syringe with an inner diameter of $0.7 \mathrm{~mm}$ blunttip needle, which connected the high voltage supply (Dongwen High Voltage Company, Tianjin, China). The syringe was fixed on a single-syringe infusion pump (749000-05, Cole-Parmer instrument company, Beijing, China) to deliver the spinning solution. The solutions were electrospun at a flow rate of $0.6 \mathrm{~mL} \mathrm{~h}^{-1}$, a high voltage of $18 \mathrm{kV}$ and a distance of $16 \mathrm{~cm}$ between the collector and needle tip. And then the resulting scaffolds were immersed in anhydrous ethanol for $15 \mathrm{~min}$ to induce a $\beta$-sheet conformational transition of SF to enhance the insolubility of scaffolds in water. The post-treated scaffolds were dried in vacuum oven at $37{ }^{\circ} \mathrm{C}$ overnight for further use. Wideangle X-ray scattering (WAXS) was performed to determine the crystallinity of post-treated scaffolds using a Bruker D8 ADVANCE X-ray diffractometer (XRD) with $\mathrm{CuK} \alpha$ radiation at $35 \mathrm{kV}$ and $30 \mathrm{~mA}$. Data were collected from $5^{\circ}$ to $55^{\circ}$ with a scanning speed of $5^{\circ} \min ^{-1}$ in a continuous mode.

The composite scaffolds embedded with MPs and MPs/ pZNF580 complexes were fabricated using alternatively layerby-layer electrospinning and electrospraying techniques. Briefly, PCL-PIBMD/SF nanofibers were firstly collected on the aluminum foil for $2 \mathrm{~h}$ with the same experimental parameters as mentioned above, and then MPs or MPs/pZNF580 suspension was electrosprayed onto the surface of PCL-PIBMD nanofibrous matrix for $1.5 \mathrm{~h}$ with applied voltage of $15 \mathrm{kV}$ and feed rate of $0.4 \mathrm{~mL} \mathrm{~h}^{-1}$. The procedure was repeated three times to complete the composite scaffolds with MPs/pZNF580 at the outermost layer.

\subsection{Characterization of electrospun scaffolds}

2.5.1. Microstructure and diameter of electrospun nanofibers. The microstructure of nanofibrous scaffold was observed by field emission scanning electron microscope (FE-SEM, Hitachi S-4800, Japan) at an accelerating voltage of $10 \mathrm{kV}$. A thin gold film was coated onto the fibrous meshes by a gold sputtering coater for $30 \mathrm{~s}$ to increase electroconductivity. The average diameter of electrospun nanofibers was measured by Image J software from SEM micrographs.

2.5.2. FT-IR measurements. Attenuated total reflection Fourier transform infrared spectroscopic analysis (ATR-FTIR, Nicolet 6700, USA) was utilized to characterize the composition and conformational changes of fibrous scaffolds with a spectral range of $4000-400 \mathrm{~cm}^{-1}$. All spectra were obtained in reflection mode on a diamond crystal by the accumulation of 32 scans with a resolution of $2 \mathrm{~cm}^{-1}$.

2.5.3. Mechanical properties measurements. The mechanical properties of electrospun membranes with rectangular shape of $10 \mathrm{~mm}$ in width and $50 \mathrm{~mm}$ in length were measured by a tensile testing instrument (WDW-02, Changchun, China) equipped with a $100 \mathrm{~N}$ load cell. The cross-head speed was $5 \mathrm{~mm} \min ^{-1}$ and the gauge length was $30 \mathrm{~mm}$. The samples were first immersed in phosphate buffer saline (PBS, pH 7.4) for $2 \mathrm{~h}$ with the purpose of mimicking the application environment and then fixed on the clamp to start tests. Five specimens were tested to calculate an average value for each sample.

2.5.4. Water contact angle measurements. The static water contact angle (WCA) of nanofibrous scaffolds was measured by the sessile drop method using a contact angle instrument (Krüss Easy Drop goniometer, Hamburg, Germany) at room temperature. The scaffolds were cut into rectangle with dimension of $10 \mathrm{~mm} \times 20 \mathrm{~mm}$. The droplet volume was set as $3 \mu \mathrm{L}$ and the instantaneous WCA was recorded within $0.5 \mathrm{~s}$ to avoid the absorption effect of water droplet by the substrate. Five measurements of each sample were used to calculate an average value.

2.5.5. Porosity testing. Square specimen was cut from the PCL-PIBMD nanofibrous scaffolds carefully and its volume $(V)$ was accurately determined by measuring its length, width and thickness. The specimen mass $(M)$ was also measured by an analytical balance with high precision of $10^{-5} \mathrm{~g}$. The apparent density $(\rho)$ of each specimen was calculate from its volume $(V)$ and mass $(M)$ by eqn (I) via multi-measurement average. The porosity $(\varepsilon)$ was calculated from $\rho$ and bulk density $\left(\rho_{0}\right)$ by eqn (III).

$$
\begin{gathered}
\rho=\frac{M}{V} \\
\rho_{0}=\frac{\rho_{\text {PCL-PIBMD }} \times \rho_{\mathrm{SF}}}{W_{\text {PCL-PIBMD }} \% \times \rho_{\mathrm{SF}}+W_{\mathrm{SF}} \% \times \rho_{\mathrm{PCL}-\mathrm{PIBMD}}}
\end{gathered}
$$




$$
\varepsilon \%=\left(1-\frac{\rho}{\rho_{0}}\right) \times 100 \%
$$

where $\rho_{\text {PCL-PIBMD }}=1.1946 \mathrm{~g} \mathrm{~cm}^{-3}, \rho_{\mathrm{SF}}=1.10 \mathrm{~g} \mathrm{~cm}^{-3}, W_{\text {PCL-PIBMD }}$ and $W_{\mathrm{SF}}$ denote the mass ratio of PCL-PIBMD and SF in the scaffold respectively.

\subsection{Biology experiments}

2.6.1. Platelet adhesion assay. The platelet adhesion of PCL-PIBMD/SF, PCL-PIBMD/SF-MPs and PCL-PIBMD/SF-MPs/ pZNF580 composite scaffolds was evaluated using previously reported method. ${ }^{45}$ The scaffolds were placed in 24-well culture plates and immersed in PBS ( $\mathrm{pH} 7.4$ ) for $12 \mathrm{~h}$, and then incubated in platelet-rich plasma (PRP, 108 platelets per $\mathrm{mL}$ ) at $37{ }^{\circ} \mathrm{C}$ for $2 \mathrm{~h}$. After rinsing with PBS several times to remove non-adherent platelets, the samples were fixed in $2.5 \mathrm{wt} \%$ glutaraldehyde in PBS for $30 \mathrm{~min}$. At last all samples were dehydrated with gradient ethanol solution and dried in vacuum oven. Platelet attachment on different scaffolds was observed using SEM to evaluate the hemocompatibility.

2.6.2. Cell culture. Human umbilical vein endothelial cells (HUVECs) were bought from Allcells Biomart (Shanghai, China) and cultured in Dulbecco's modified Eagle medium (DMEM, Gibco, USA) supplemented with 10\% fetal bovine serum (FBS, Gibco), $100 \mu \mathrm{g} \mathrm{mL}{ }^{-1}$ penicillin and $100 \mu \mathrm{g} \mathrm{mL}^{-1}$ streptomycin in an incubator $\left(37^{\circ} \mathrm{C}, 5 \% \mathrm{CO}_{2}\right)$. Fresh culture medium was changed every other day until the HUVECs reached about $90 \%$ confluence, and then the cells were trypsinized to subculture according to the standard procedure. The cell densities during all experiments were accurately determined by a hematocytometer and all specimens were sterilized before cell studies.

2.6.3. CCK-8 assay. Cell counting kit assay (CCK-8, Beyotime, China) was utilized to evaluate the cell proliferation of HUVECs on sandwich-like PCL-PIBMD/pZNF580 scaffolds at time points of 1,3 and 5 days. HUVECs were seed at a density of $1 \times 10^{4}$ cells per well onto the scaffolds in 96-well culture plate at $37{ }^{\circ} \mathrm{C}$ in $5 \% \mathrm{CO}_{2}$. A $20 \mu \mathrm{L} \mathrm{CCK}-8$ solution was added to each well and incubated for 4 hours at predetermined points (2 days). Then $150 \mu \mathrm{L}$ mixture was transferred into another 96-well culture plate for measurement. The optical density (OD) of each well was measured using a microplate reader (Bio-Rad, IMARK $^{\mathrm{TM}}$ ) at a test wavelength of $450 \mathrm{~nm}$. The OD values of each well were used to represent the survival/proliferation of HUVECs. All results were obtained from three parallel measurements.

2.6.4. FDA staining assay. Fluorescein diacetate (FDA) staining assay was used to investigate the adhesion, spread and proliferation of HUVECs. The cell-seeded scaffolds were prepared as above, and then the samples were analysed by FDA assay method at predetermined time points (1, 3 and 5 days). Briefly, FDA solution ( $5 \mathrm{mg} \mathrm{mL}^{-1}$ in acetone) were added to 96 well culture plate to stain the HUVECs on the scaffolds for $30 \mathrm{~min}$ and then the sample were washed with D-Hanks solution thrice. Afterwards, the adhered HUVECs were stained with FDA and images were obtained using the inverted fluorescent Microscope (Olympus CKX41, Tokyo, Japan). The cell densities (number of cells per $\mathrm{mm}^{2}, \mathrm{~N} \mathrm{~mm}^{-2}$ ) on the composite scaffolds were determined by Image-Pro Plus software according to three random fluorescence images.

2.6.5. Cell adhesion assay. Composite scaffolds were sterilized and cut to fit the size of 96-well plate first. Then the scaffolds were put into 96-well culture plates and HUVECs were trypsinized, counted and plated at a density of $1 \times 10^{4}$ cells per well onto the surface of scaffolds. The culture condition was set as $37{ }^{\circ} \mathrm{C}$ and $5 \% \mathrm{CO}_{2}$. After 1,3 and 5 days of cell culture, the composite scaffolds were harvested, and rinsed thrice with PBS to remove non-adherent cells and fixed with $2.5 \%$ glutaraldehyde at $4{ }^{\circ} \mathrm{C}$ for $2 \mathrm{~h}$. The scaffolds were dehydrated using gradient ethanol solution from $50 \%$ to $100 \%$ in the concentration difference of $10 \%$ for $10 \mathrm{~min}$, and then lyophilized. SEM was used to evaluate cell morphology and adhesion to the scaffolds at an accelerating voltage of $10 \mathrm{kV}$. The coverage ratios of HUVECs on the composite scaffolds were calculated from three random SEM images using Image $\mathrm{J}$ software.

2.6.6. In vivo subcutaneous implantation assay. Animal studies were conducted in the department of physiology and pathophysiology, Logistics University of Chinese People's Armed Police Force, which has an SPF fully enclosed animal feeding room and standard animal experimental facilities. All animal experiments were performed in compliance with the Guidelines on the Humane Treatment of Laboratory Animals (Ministry of Science and Technology of China, 2006) and approved by the Animal Care Committee and Laboratory Animal Administration Rules of Logistics University of Chinese People's Armed Police Force (Tianjin, China). Six SpragueDawley rats (weight about $125 \mathrm{~g}$, aged from 2 to 3 months) were used for this study and randomly divided into three groups (two rats per group). Pre-sterilized PCL-PIBMD/SF, PCL-PIBMD/ SF-MPs and PCL-PIBMD/SF-MPs/pZNF580 composite scaffolds with diameter of $6 \mathrm{~mm}$ were subcutaneously implanted in both sides of the back. After 2 weeks, the samples with surrounding tissue were excised to evaluate the tissue response. The asobtained tissue samples were fixed in 10\% formaldehyde for 2 days and then dehydrated in gradient ethanol, saturated with xylene, imbedded in paraffin for further histological analysis including hematoxylin and eosin (H\&E) staining and Masson's trichrome staining.

\subsection{Statistical analysis}

All data were represented as mean \pm SD (standard deviation of the mean value) at least three parallel measurements and compared by using one-way ANOVA tests. A $p$-value less than 0.05 indicated statistically significant differences between groups.

\section{Results and discussion}

\subsection{Morphology and microstructure of PCL-PIBMD/SF scaffolds}

Many electrospinning parameters can affect the morphology and dimension of nanofibers, such as the distance from tip to collector, solvent, applied voltage and flow rate. Usually, closer distance between the tip and collector yields smaller fibers. 
Meanwhile, it is worth noting that a minimum distance is required to guarantee the complete evaporation of solvent from the electrospun nanofibers, otherwise nanofibers with beads may occur when the distance is too close or too far. The electrospinning solvent should possess high volatility, low boiling point and high vapor pressure. The solvent plays a significant role in the formation of nanostructure because it affects the surface tension and phase separation. The SEM micrographs of PCL-PIBMD/SF blend scaffolds with different weight ratio are shown in Fig. 1. No nodules were observed in all blend scaffolds, and the nanofibers were smooth and continuous when electrospinning was carried out at a flow rate of $0.6 \mathrm{~mL} \mathrm{~h}^{-1}$, a high voltage of $18 \mathrm{kV}$ and a distance of $16 \mathrm{~cm}$ between the collector and needle tip. These results indicated that the selected parameters were proper and the solvent had evaporated in electrospinning process. The average diameter of nanofibers decreased from $573.8 \pm 18.3 \mathrm{~nm}$ to $285.1 \pm 23.3 \mathrm{~nm}$ with SF content increasing from 0 to $90 \%$, because the electrical conductivity of electrospinning solution increased along with the addition of negatively charged SF. ${ }^{46}$ The high conductivity induced high elongation and drawing force on the jet, which benefits to form the nanofibers with small diameter. For example, when SF content was $10 \%$, the average diameter was $516.8 \pm 12.9 \mathrm{~nm}$; when SF content increased to $70 \%$, the average diameter decreased to $340.5 \pm 9.1 \mathrm{~nm}$ accordingly.

\section{2. $\quad$ ATR-FTIR analysis of PCL-PIBMD/SF scaffolds}

ATR-FTIR analysis was utilized to characterize the composition and functional groups of PCL-PIBMD/SF blend scaffolds. As shown in Fig. 2, the absorption signal at $3288 \mathrm{~cm}^{-1}$ was the characteristic peak of secondary amide (-NH-) stretching vibration in the IBMD segment. The absorption peaks at 2952 and $2870 \mathrm{~cm}^{-1}$ were attributed to the stretching vibration of saturated $\mathrm{C}-\mathrm{H}$ bonds in the methyl $\left(-\mathrm{CH}_{3}\right)$ and methylene $\left(-\mathrm{CH}_{2}-\right)$. Carbonyl bond $(-\mathrm{C}=\mathrm{O})$ is the most easily distinguished group in a spectrum. ${ }^{47}$ In the spectrum of PCL-PIBMD/ SF scaffolds, the strong absorption at $1731 \mathrm{~cm}^{-1}$ referred to the $-\mathrm{C}=\mathrm{O}$ stretching vibration, and correspondingly the signals at 1245 and $1162 \mathrm{~cm}^{-1}$ resulted from $-\mathrm{C}=\mathrm{O}$ bending vibration and $-\mathrm{C}-\mathrm{O}-$ stretching vibration, respectively. Moreover, PCLPIBMD/SF scaffolds exhibited two strong peaks at 1650 and $1532 \mathrm{~cm}^{-1}$, which are the typical absorption peaks of SF with $\beta$ sheet conformation. The absorption peak at $1162 \mathrm{~cm}^{-1}$ became weaker gradually with the increase of SF content in the PCLPIBMD/SF blend scaffolds.

\subsection{Mechanical properties of PCL-PIBMD/SF scaffolds}

Tissue engineering vascular scaffolds need to possess sufficient mechanical strength to meet the demands in clinical application. Artificial vascular scaffolds with inadequate mechanical
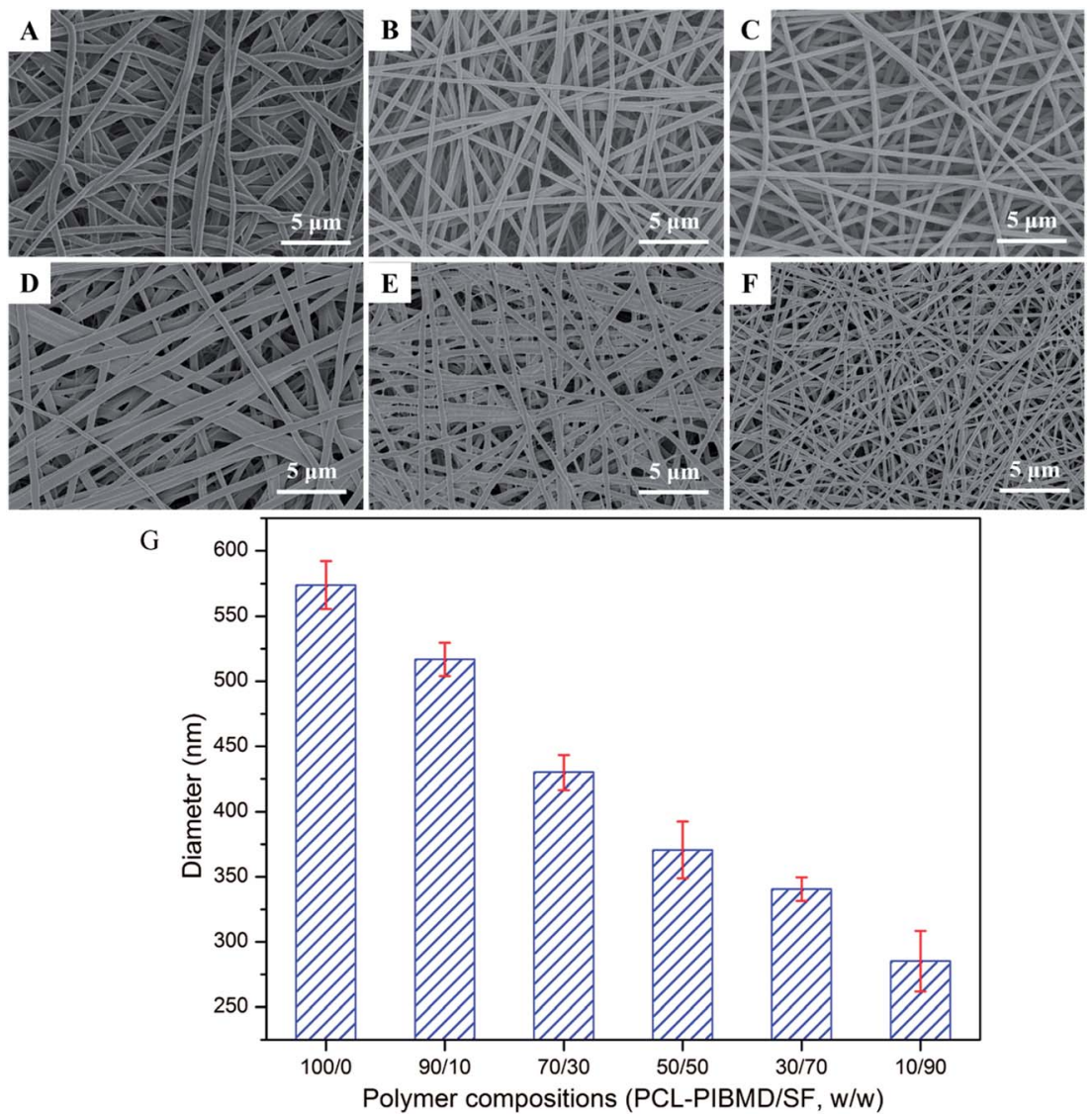

Fig. 1 SEM micrographs of electrospun PCL-PIBMD/SF nanofibrous scaffolds with various PCL-PIBMD/SF weight ratio: (A) 100/0, (B) 90/10, (C) 70/30, (D) 50/50, (E) 30/70, (F) 10/90 and (G) average diameter of electrospun PCL-PIBMD/SF nanofibrous scaffolds. (Error bars represent mean $\pm \mathrm{SD}$.) 


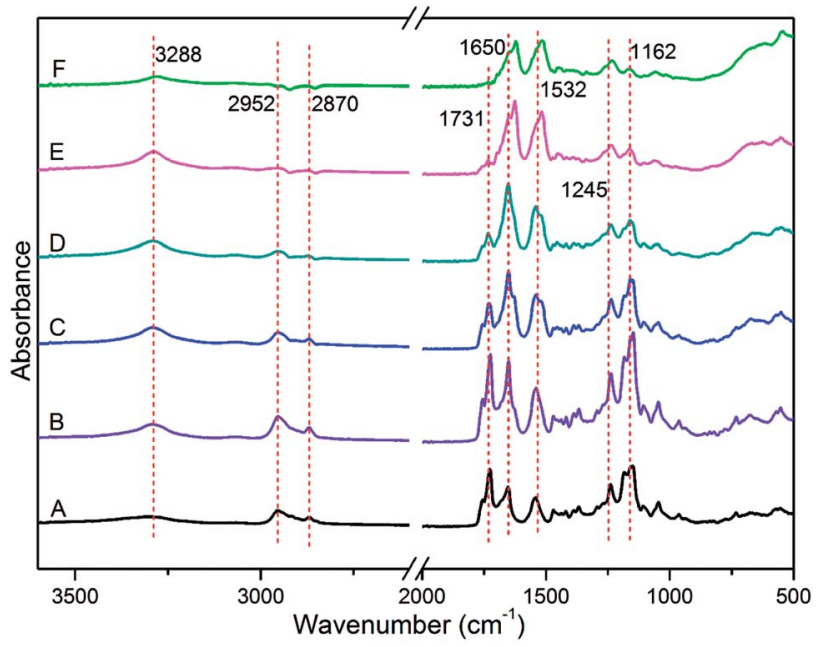

Fig. 2 ATR-FTIR of electrospun PCL-PIBMD/SF nanofibrous scaffolds with various PCL-PIBMD/SF weight ratio: (A) 100/0, (B) 90/10, (C) 70/ 30, (D) 50/50, (E) 30/70, (F) 10/90.

strength could not bear blood flow force after vascular transplantation, which likely leads to the failure of vascular surgery and threatens the lives of patients. It is well known that the tensile strength of fresh carotid arteries is about 1.76-2.64 MPa and elongation at break is about $110-200 \% .^{48} \mathrm{Fig} .3$ shows the tensile strength and elongation at break of PCL-PIBMD/SF scaffolds with different weight ratios. Along with increasing SF content in PCL-PIBMD/SF blend scaffolds, the tensile strength of scaffolds decreased from $7.94 \pm 0.09$ to $1.63 \pm$ $0.17 \mathrm{MPa}$ and elongation at break varied from $169 \pm 30 \%$ to 12 $\pm 3 \%$, respectively. When the weight ratio of SF was $10 \%$, the tensile strength and elongation at break of scaffolds were found to be $7.05 \pm 0.34 \mathrm{MPa}$ and $210 \pm 21 \%$, which was suitable for vascular scaffolds.

\subsection{Surface wettability of PCL-PIBMD/SF scaffolds}

Surface wettability is regarded as one of the most important surface properties affecting the biological response of an implanted prosthesis, including cell attachment, spreading, growth and proliferation. WCA measurements were performed to investigate the surface wettability of PCL-PIBMD/SF scaffolds. As shown in Fig. 4, PCL-PIBMD nanofibrous scaffolds showed the highest WCA value of $132.8 \pm 1.9^{\circ}$ among all scaffolds. It is evident that the WCA values decreased with increasing SF content in PCL-PIBMD/SF blend scaffolds. For example, WCA value of the scaffolds with the weight ratio of 90/10 was $123.1 \pm$ $1.3^{\circ}$. When SF content reached to $90 \%$, the scaffolds merely exhibited moderately hydrophilic characteristics with a WCA value of $82.7 \pm 4.5^{\circ}$. However, it is noteworthy that the decline was not so remarkable as we expected. The possible reason is that ethanol treatment resulted in the increase of parallel folding crystalline structure of SF in the scaffolds, which weakened the hydrophilicity. WAXS results confirmed this (Fig. 5). The XRD patterns of PCL-PIBMD/SF blend scaffolds exhibited the characteristic peaks related to PCL-PIBMD and SF.
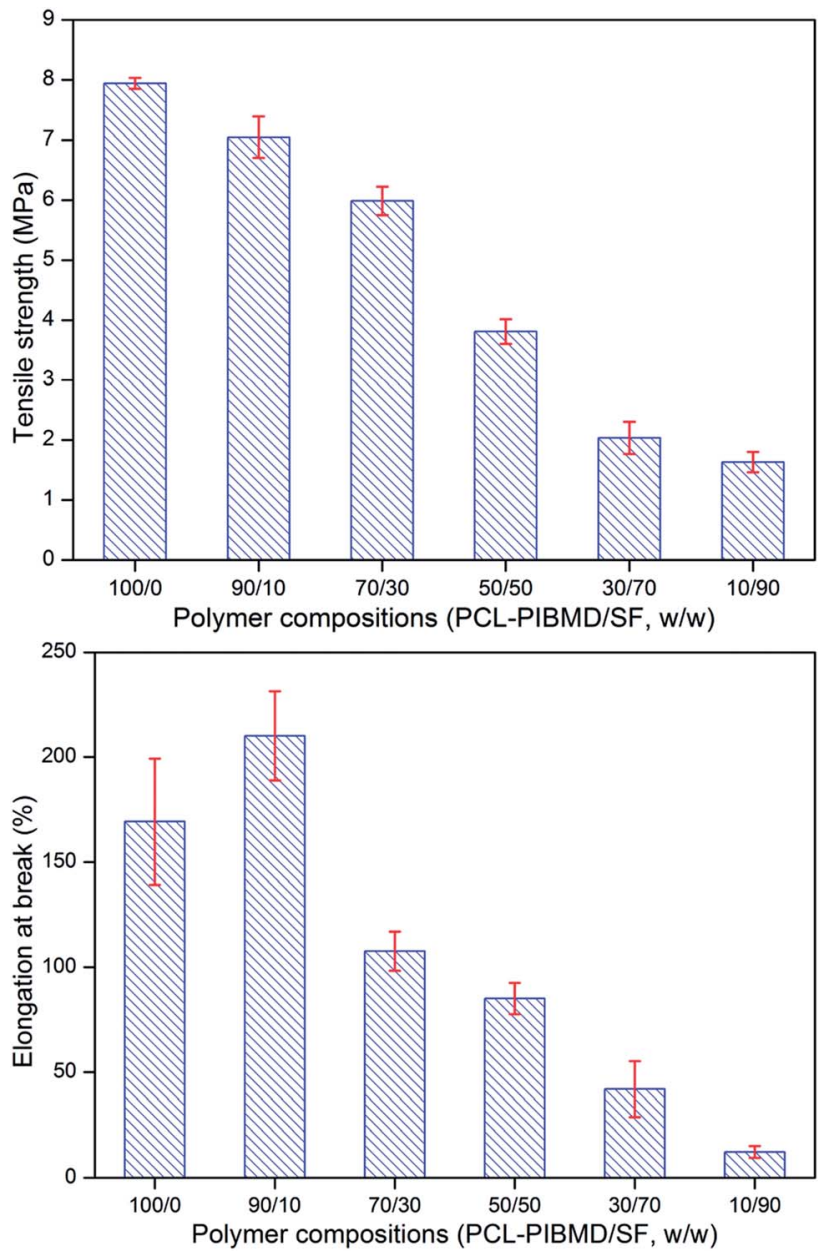

Fig. 3 Tensile strength and elongation at break of electrospun PCLPIBMD/SF nanofibrous scaffolds with various PCL-PIBMD/SF weight ratio. (Error bars represent mean $\pm \mathrm{SD}$.)

The crystallinity of PCL-PIBMD/SF scaffolds with the weight ratio from $100 / 0$ to $10 / 90$ was $37 \%, 43 \%, 28 \%, 25 \%, 16 \%$ and $11 \%$ respectively, showing a decreasing trend with the increase

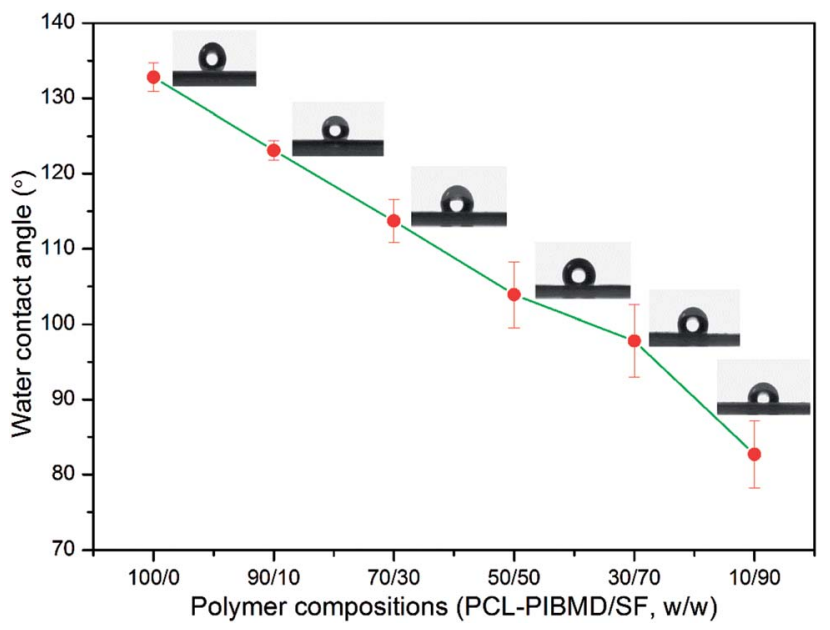

Fig. 4 Water contact angle of electrospun PCL-PIBMD/SF nanofibrous scaffolds with various PCL-PIBMD/SF weight ratio. (Error bars represent mean $\pm \mathrm{SD}$.) 


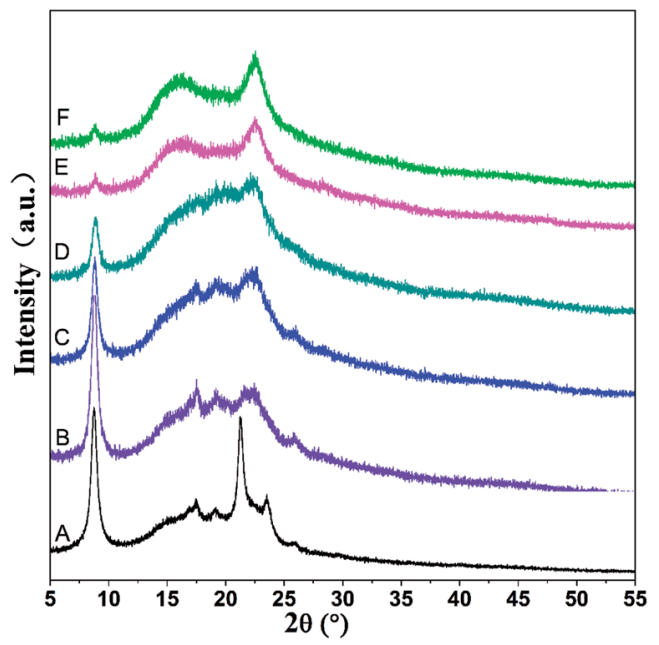

Fig. 5 X-ray diffraction patterns of ethanol-treated electrospun PCLPIBMD/SF nanofibrous scaffolds with various PCL-PIBMD/SF weight ratio: (A) 100/0, (B) 90/10, (C) 70/30, (D) 50/50, (E) 30/70, (F) 10/90.

of SF content. The crystalline region of PCL-PIBMD and SF is incompatible and prone to phase separation. SF can hinder PCL-PIBMD to form crystalline structure. The intensity of the peak at $22.5^{\circ}$ corresponding to SF became strong with the increase of SF content, while the intensity of crystallization peak at $8.8^{\circ}$ related to PCL-PIBMD became weak. SF can change the crystallinity and wettability of scaffold materials.

\subsection{Porosity of PCL-PIBMD/SF scaffolds}

The high porosity of a scaffold is of significance to complete efficient cell infiltration. ${ }^{49}$ A low porosity may limit cell migration in a biodegradable scaffold, which is not suitable for tissue engineering. ${ }^{50}$ As shown in Fig. 6, the porosity of scaffolds clearly exhibited a decreasing tendency along with SF increase. The porosity was $75.6 \pm 3.0 \%, 70.1 \pm 2.1 \%, 64.8 \pm 1.0 \%, 58.3 \pm$

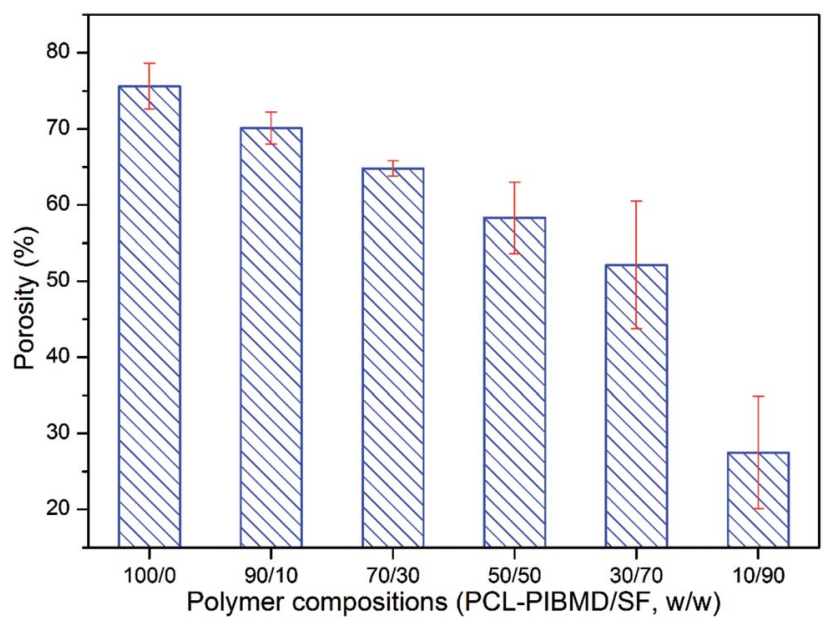

Fig. 6 Porosity of electrospun PCL-PIBMD/SF nanofibrous scaffolds with various $P C L$-PIBMD/SF weight ratio. (Error bars represent mean \pm SD.)
4.7\%, $52.1 \pm 8.4 \%$ and $27.5 \pm 7.4 \%$ for PCL-PIBMD/SF scaffolds with weight ratios of 100/0, 90/10, 70/30, 50/50, 30/70, 10/90, respectively. The results were consistent with previous studies, which demonstrated that the low porosity of a scaffold could be formed by increasing the fiber packing density during electrospinning. ${ }^{51,52}$ Small-diameter nanofibers could result in a high packing density, thus easily producing a scaffold with low porosity, while large-diameter nanofibers formed scaffolds with high porosity. For example, PCL-PIBMD with diameter of 573.8 $\pm 18.3 \mathrm{~nm}$ had a high porosity of $75.6 \pm 3.0 \%$. Considering the above properties, we chose PCL-PIBMD/SF scaffolds with weight ratio of $90 / 10$ for further study.

\subsection{Morphology of pZNF580 complexes-containing composite scaffolds}

MPs and MPs/pZNF580 complexes were embedded into PCLPIBMD/SF scaffolds through alternatively layer-by-layer electrospinning and electrospraying techniques. TEM was used to observe the morphology of as-prepared MPs and MPs/pZNF580 complexes, and the images were shown in Fig. 7. They exhibited round-shape with size distribution of $106 \pm 28 \mathrm{~nm}$ and $98 \pm$ $28 \mathrm{~nm}$. Furthermore, SEM was used to elucidate whether MPs were loaded into scaffolds or not, and whether the layer structure existed in the scaffolds. The surface and cross-section micrographs of scaffolds were shown in Fig. 8. Compared with blank scaffold (Fig. 8A1), the surface of scaffolds embedded with MPs or MPs/pZNF580 complexes had a lot of granular aggregates consisted of regular spheres at a larger magnification (Fig. 8B1 and C1). Additionally, according to the cross-section micrographs, the PCL-PIBMD/SF-MPs (Fig. 8B2) and PCL-PIBMD/SF-MPs/pZNF580 (Fig. 8C2) scaffolds had sandwich-like lay-structure. The lay-structure also contained many round-shape microparticles with average diameter of $79 \mathrm{~nm}$ in line with the size of as-prepared MPs and MPs/ pZNF580. Collectively, these results demonstrated that MPs and MPs/pZNF580 had been successfully embedded into PCLPIBMD/SF scaffolds.

\subsection{Platelet adhesion test}

For blood-contact materials, platelet adhesion plays an important role in foreign-body reaction and thrombosis formation. ${ }^{53}$ Generally, the low platelet adhesion, the better hemocompatibility of biomaterial surface. In order to evaluate the hemocompatibility of PCL-PIBMD/SF-MPs/pZNF580 scaffolds, platelet adhesion test was conducted with PCL-PIBMD/SF as a control. The platelet adhesion on the scaffolds was observed through SEM (Fig. 9). The results indicated that all experimental groups had low platelet adhesion, but the PCL-PIBMD/SF scaffold exhibited relatively higher platelet adhesion than PCLPIBMD/SF-MPs and PCL-PIBMD/SF-MPs/pZNF580 after incubating with PRP for $2 \mathrm{~h}$. PCL-PIBMD/SF-MPs and PCL-PIBMD/ SF-MPs/pZNF580 scaffolds had barely platelet adhesion, which most likely resulted from hydrophilic PEG in MPs to inhibit platelet adhesion. It was also confirmed by the WCA measurement, in which PCL-PIBMD/SF-MPs and PCL-PIBMD/ 

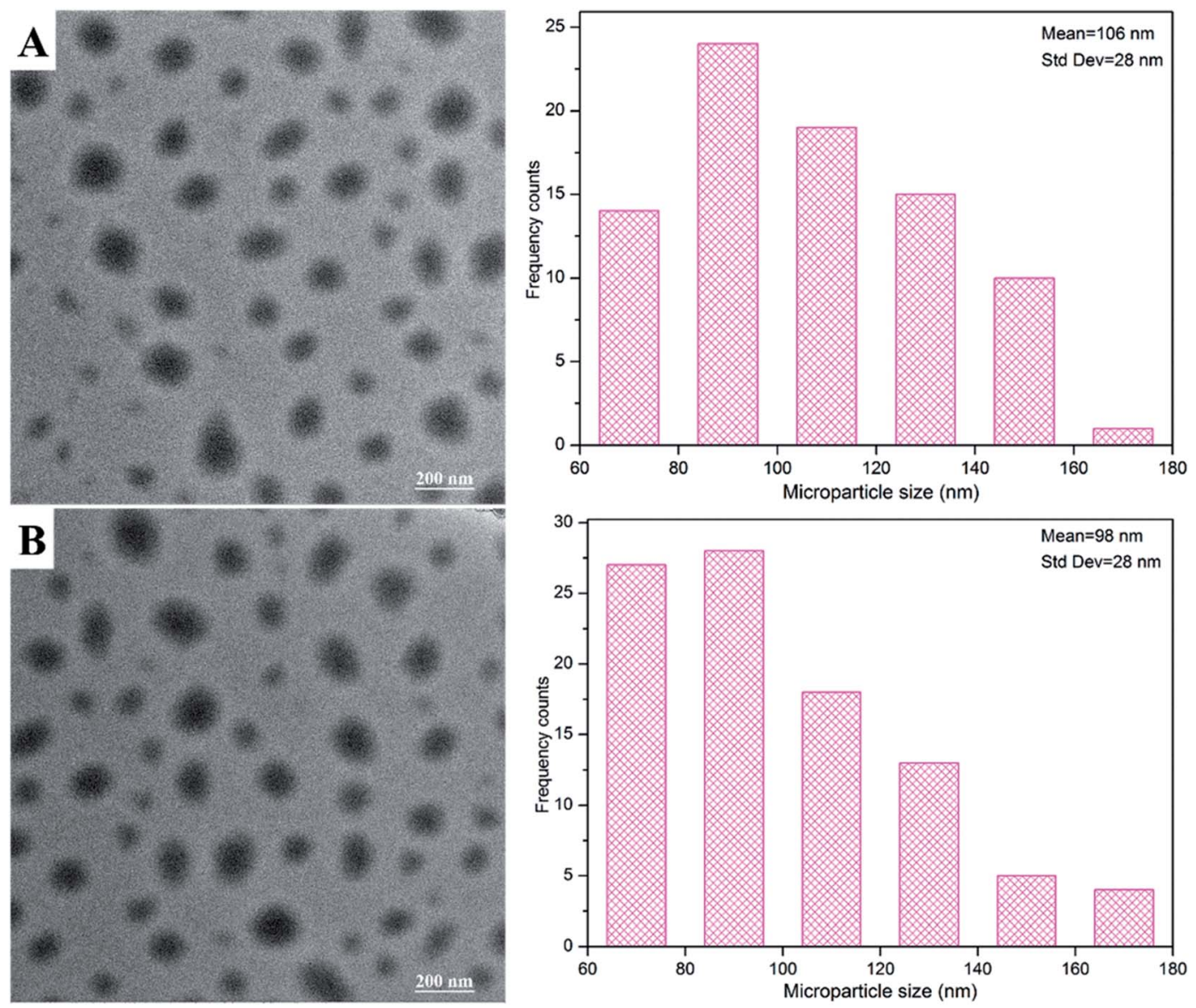

Fig. 7 TEM images and size distribution of as-prepared MPs (A) and MPs/pZNF580 complexes (B) composed of mPEG-b-PLGA-g-PEI-REDV and mPEG- $b$-PLGA-g-PEI-REDV/pZNF580, respectively.

SF-MPs/pZNF580 scaffold had lower WCA value than blank PCLPIBMD/SF scaffold (Fig. 9).

\subsection{Proliferation of HUVECs on MPs/pZNF580-loaded scaffolds}

In order to determine cell response to MPs/pZNF580-loaded scaffolds, HUVECs were seeded on these scaffolds, and the cell proliferation was evaluated by CCK-8 assay on day 1, 3 and 5 , respectively. Cells without treatment were used as a control. As shown in Fig. 10, proliferation increased along with the culture time, indicating cell proliferation proceeded normally. On day 1 , cell proliferation in PCL-PIBMD, PCL-PIBMD/SF-MPs and PCL-PIBMD/SF-MPs/pZNF580 scaffold groups did not have significant difference. However, on day 3 and 5, cell proliferation in the PCL-PIBMD/SF-MPs/pZNF580 scaffold group was evidently higher than those of the PCL-PIBMD and PCL-PIBMD/ SF-MPs scaffold groups. It was noteworthy that cell proliferation in PCL-PIBMD/SF-MPs/pZNF580 scaffold group was almost similar to the control on day 5 , even though the slight cytotoxicity of scaffold materials could inhibit cell proliferation to some degree. The abovementioned results demonstrated that the scaffolds containing MPs/pZNF580 complexes could promote cell proliferation of HUVECs in accord with previous studies. ${ }^{21-23}$

\subsection{Adhesion, spreading and migration of HUVECs on MPs/ pZNF580-loaded scaffolds}

The surface properties of scaffolds notably influence the adhesion, spreading and migration of HUVECs, and eventually determining whether the scaffold transplantation is successful or not. $^{54,55}$ The cellular growth behavior of HUVECs on MPs/ pZNF580-loaded scaffolds was investigated through FDA fluorescence staining assay and cell spreading assay on different culture time (1, 3 and 5 day). The fluorescence images and SEM micrographs as well as the corresponding quantitative results were presented in Fig. 11 and 12, respectively. It was found that the number of HUVECs increased with culture time on three different scaffolds in the fluorescence images. Furthermore, compared with the other three groups, HUVECs on MPs/ pZNF580-loaded scaffolds exhibited high growth rate, which could be attributed to the high transfection ability of plasmid complexes embedded in the composite scaffolds. The PCLPIBMD/SF-MPs scaffolds without pZNF580 also presented a slightly higher cell number than blank PCL-PIBMD/SF scaffolds due to its moderate hydrophilicity resulted from PEG. For example, the cell densities of PCL-PIBMD/SF-MPs and PCLPIBMD/SF-MPs/pZNF580 scaffolds were $265 \pm 6 \mathrm{~N} \mathrm{~mm}^{-2}$ and $358 \pm 10 \mathrm{~N} \mathrm{~mm}^{-2}$ on day 3 , which were obviously higher than that of blank PCL-PIBMD/SF scaffold $\left(180 \mathrm{~N} \mathrm{~mm}^{-2}\right)$. On day 5 , 

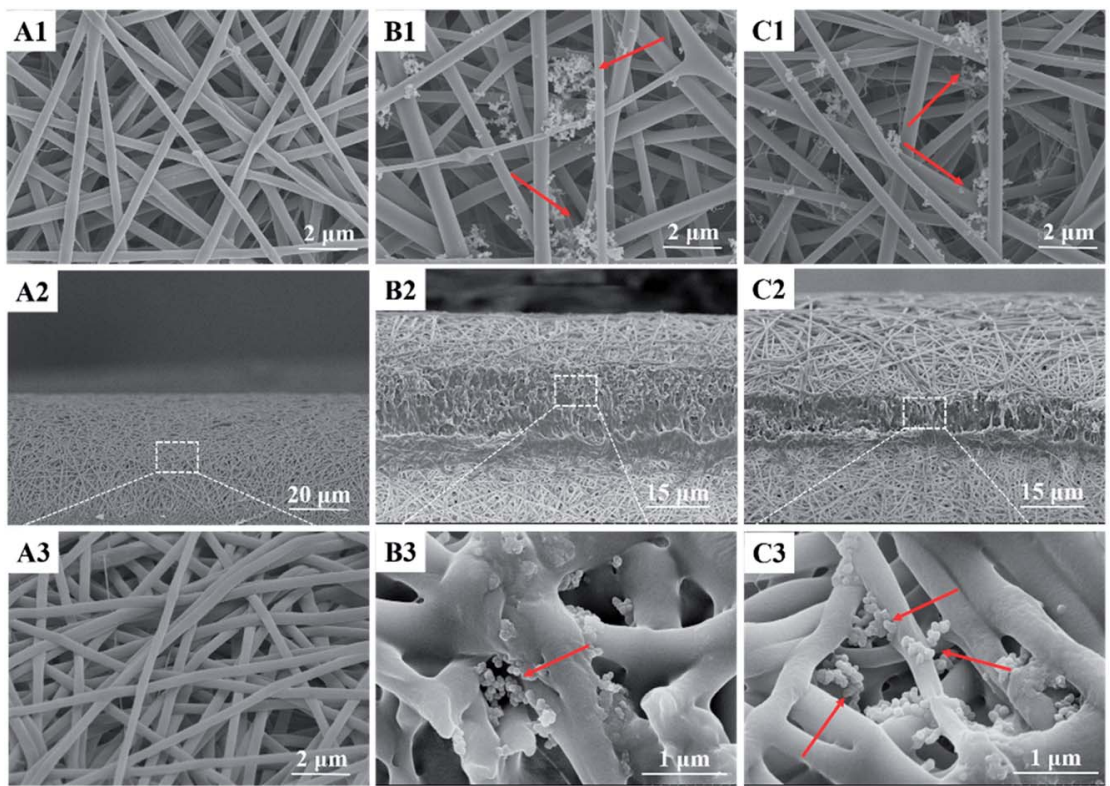

Fig. 8 SEM micrographs of the surface and cross-section of composite scaffolds: the surface morphology of (A1) PCL-PIBMD/SF, (B1) PCLPIBMD/SF-MPs and (C1) PCL-PIBMD/SF-MPs/pZNF580 scaffold at a magnification of $10000 \times$; the cross-sectional morphology of (A2) PCLPIBMD/SF $(1000 \times)$ ), (B2) PCL-PIBMD/SF-MPs and (C2) PCL-PIBMD/SF-MPs/pZNF580 scaffold (1500x), and larger magnification of the inset boxes $(A 3,10000 \times ; B 3$ and $C 3,30000 \times)$.
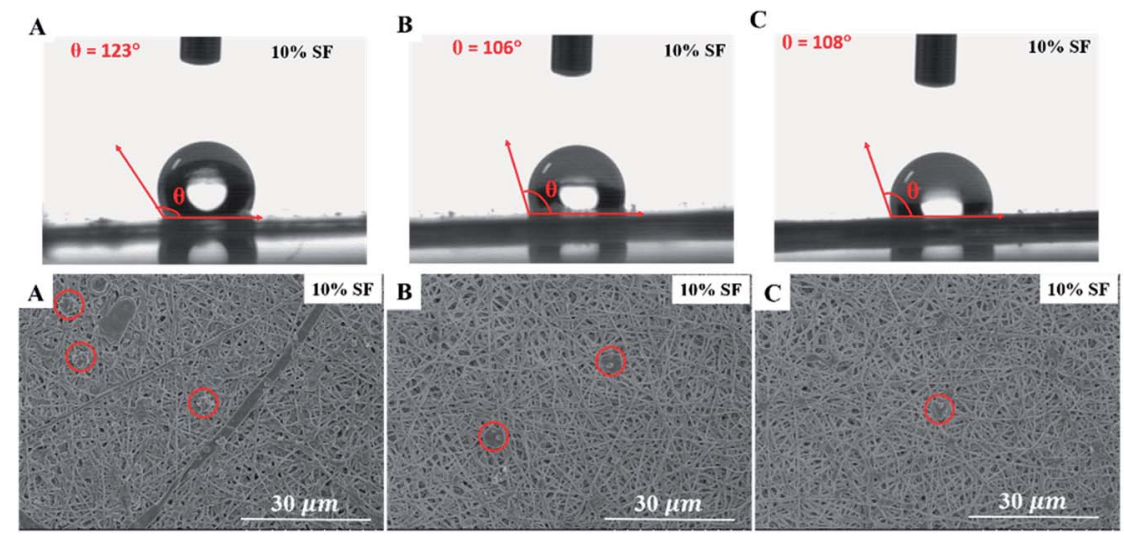

Fig. 9 Water contact angle and SEM micrographs of platelet adhesion on composite scaffolds: (A) PCL-PIBMD/SF scaffold; (B) PCL-PIBMD/SFMPs scaffold; and (C) PCL-PIBMD/SF-MPs/pZNF580 scaffold. (Red circles represent the location of platelets in the viewing zone.)

HUVECs on the PCL-PIBMD/SF-MPs/pZNF580 scaffolds totally overspread the viewing zone. The results of cell spreading assay were similar to fluorescence assay. HUVECs adhered, grew and migrated on these scaffolds, and covered more area along with culture time. The coverage ratio of PCL-PIBMD/SF, PIBMD/SFMPs and PCL-PIBMD/SF-MPs/pZNF580 scaffolds on day 3 were $33 \pm 2 \%, 46 \pm 1 \%$ and $66 \pm 3 \%$, respectively. In addition, HUVECs spread and migrated most quickly on the PCL-PIBMD/ SF-MPs/pZNF580 scaffold surface, and nearly covered full area of these scaffolds on day 5. FDA fluorescence staining assay and cell spreading assay both confirmed that MPs/pZNF580-loaded scaffolds could effectively promote adhesion, spreading and migration of HUVECs.

\subsection{In vivo biocompatibility of MPs/pZNF580-loaded scaffolds}

Tissue compatibility is a substantial factor for successful transplant of vascular scaffolds. The inflammatory events induced by implanted biomaterials are modulated by several kinds of inflammatory cells, such as macrophages, lymphocytes and neutrophils. Unexpected tissue response may compromise the safety, biofunction and performance of implanted devices via complex interactions with biological component. ${ }^{56}$ Thus, vascular scaffolds with slighter tissue responses are anticipated as valuable candidates for clinic treatment. The tissue response of composite scaffolds was evaluated by subcutaneous implantation in Sprague-Dawley rats for 2 weeks. The representative micrographs of H\&E staining and Masson's trichrome 


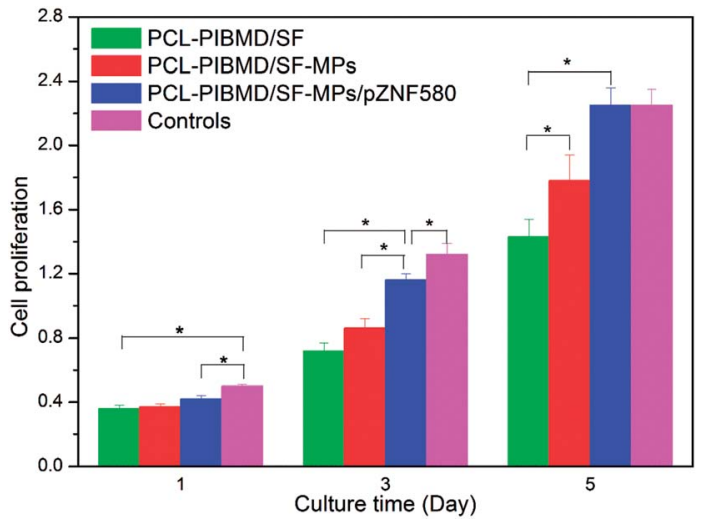

Fig. 10 CCK-8 value of HUVECs cultured on PCL-PIBMD/SF, PCLPIBMD/SF-MPs and PCL-PIBMD/SF-MPs/pZNF580 scaffold for 1, 3, and 5 days. Cells without treatment served as a control. (Error bars represent mean $\pm \mathrm{SD}$. * denote statistically significant difference between pairs.)

staining are represented in Fig. 13. Although all implanted scaffolds exhibited the infiltration of inflammatory cells with different degrees, PCL-PIBMD/SF, PCL-PIBMD/SF-MPs and PCL-
PIBMD/SF-MPs/pZNF580 composite scaffolds did not induce obvious tissue toxicity and tissue response. For PCL-PIBMD/SFMPs/pZNF580 composite scaffolds, few granulations composed of inflammatory cells were observed compared with PCLPIBMD/SF and PCL-PIBMD/SF-MPs scaffolds according to the micrographs of H\&E staining, indicating a slight inflammatory response. In addition, as shown in the micrographs of Masson's trichrome staining, a fibrous capsule $(19 \pm 4 \mu \mathrm{m})$ generated at the tissue/material interface for PCL-PIBMD/SF-MPs/pZNF580 composite scaffold, which was evidently thinner than those of PCL-PIBMD/SF $(27 \pm 6 \mu \mathrm{m})$ and PCL-PIBMD/SF-MPs $(61 \pm 11$ $\mu \mathrm{m})$ scaffolds. Fibrous capsules usually separate the implanted biomedical devices from normal host tissue, which may hamper the performance of devices and induce a risk of infection at the implanted site. In general, the thinner fibrous capsule implies slighter tissue response. ${ }^{57}$ The introduction of plasmid complexes into scaffolds attenuated the granulation and fibrous capsule formation, which possibly resulted from the expression of pZNF580 plasmids via an NF- $\kappa$ B signalling pathway. ${ }^{58}$ Overall, PCL-PIBMD/SF-MPs/pZNF580 composite scaffold had mitigated inflammatory reaction around the implantation site, and

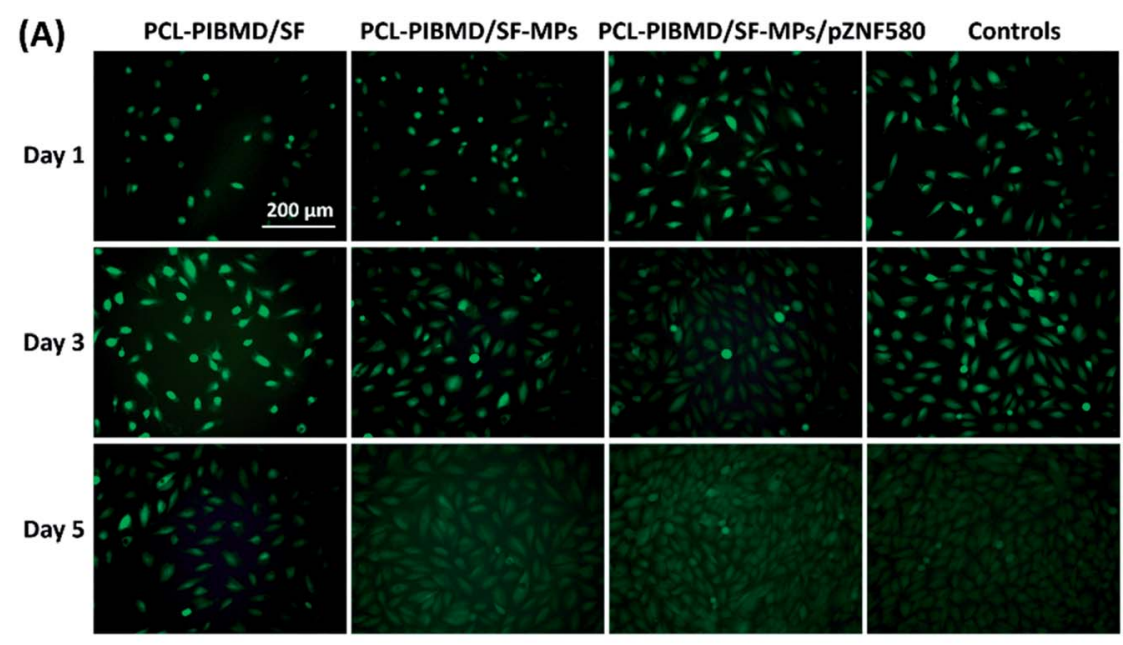

(B)



Fig. 11 Fluorescence micrographs (A) and cell density (B) of HUVECs cultured on PCL-PIBMD/SF, PCL-PIBMD/SF-MPs and PCL-PIBMD/SF-MPs/ pZNF580 scaffold for 1,3 , and 5 days. Cells without treatment served as a control in figure. (Error bars represent mean \pm SD. * denote statistically significant difference between pairs.) 


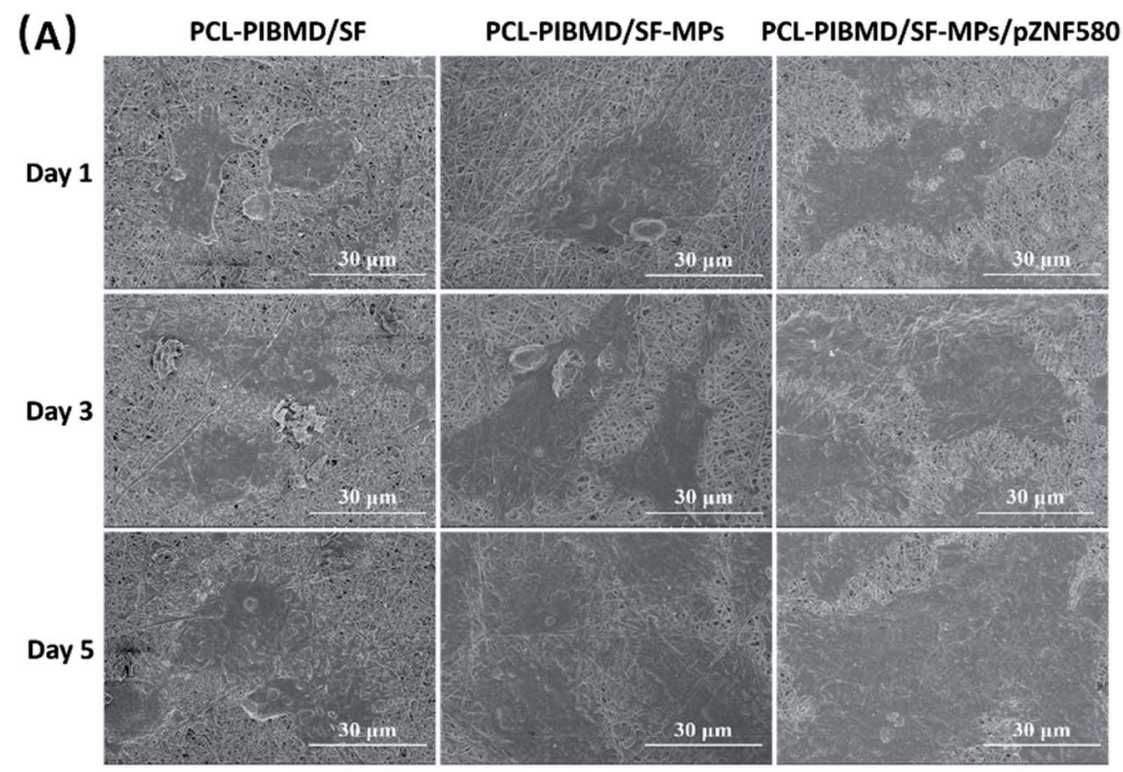

(B)

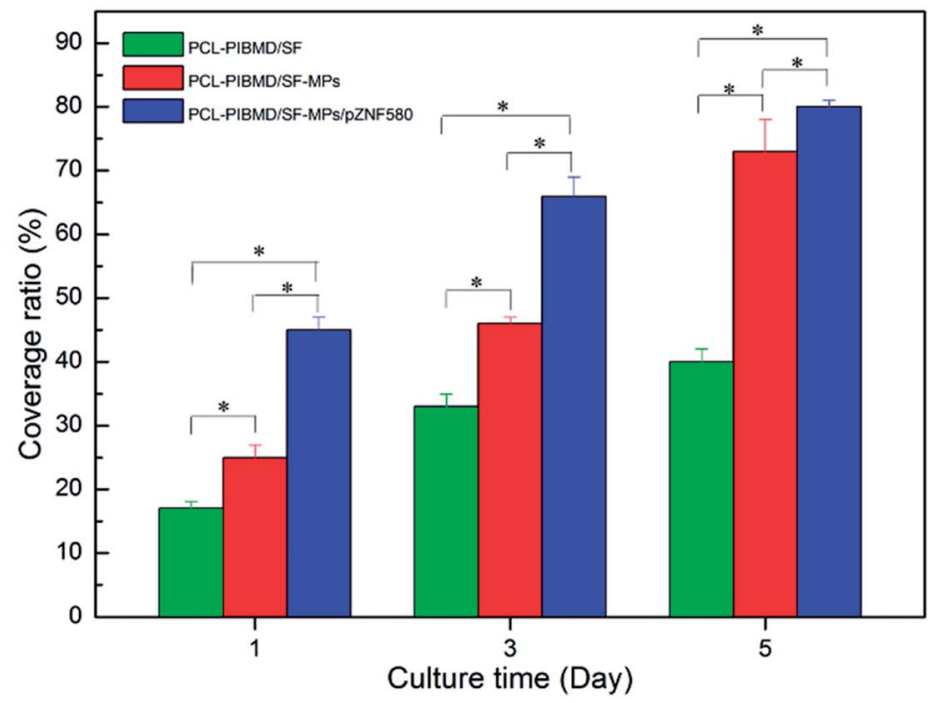

Fig. 12 SEM micrographs (A) and coverage ratio (B) of HUVECs cultured on PCL-PIBMD/SF, PCL-PIBMD/SF-MPs and PCL-PIBMD/SF-MPs/ pZNF580 scaffold for 1,3 , and 5 days. (Error bars represent mean \pm SD. * denote statistically significant difference between pairs.)

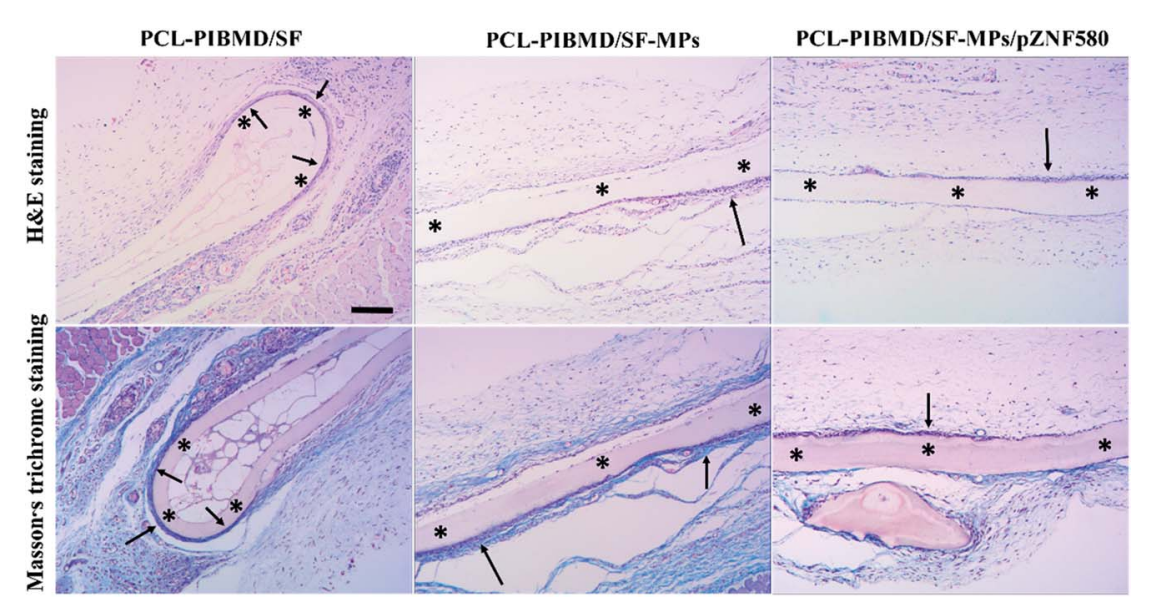

Fig. 13 H\&E staining and Masson's trichrome staining of subcutaneous tissue around PCL-PIBMD/SF, PCL-PIBMD/SF-MPS and PCL-PIBMD/SFMPs/pZNF580 scaffold after 2 weeks implantation. * represents the implant site of scaffold. Scale bar: $200 \mu \mathrm{m}$. 
could serve as an excellent and acceptable candidate of vascular scaffolds for demanding long-term performance.

\section{Conclusions}

Sandwich-like composite scaffolds delivering plasmid complexes with the purpose of maintaining the complete structure and functionality of plasmid complexes were successfully prepared using alternatively layer-by-layer electrospinning and electrospraying techniques. The layer composed of plasmid complexes could evidently help plasmid complexes maintain their own structure stability and function integrity in the scaffolds. The composite scaffolds containing plasmid complexes not only possessed superior mechanical properties, moderate surface wettability, high porosity, good cytocompatibility, good hemocompatibility and excellent histocompatibility, but also promoted the proliferation, adhesion, spreading and migration of HUVECs. Thus, these composite scaffolds could be an ideal platform combining gene therapy and tissue regeneration for application in vascular tissue engineering.

\section{Conflicts of interest}

There are no conflicts of interest to declare.

\section{Acknowledgements}

This project was supported by State Key Project of Research and Development (Grant 2016YFC1100300), the National Natural Science Foundation of China (Grants 31370969 and 51673145), International Science \& Technology Cooperation Program of China (Grant 2013DFG52040).

\section{References}

1 S. Pashneh-Tala, S. MacNeil and F. Claeyssens, The tissueengineered vascular graft-past, present, and future, Tissue Eng., Part B, 2015, 22, 68-100.

2 A. Hasan, A. Memic, N. Annabi, et al., Electrospun scaffolds for tissue engineering of vascular grafts, Acta Biomater., 2014, 10, 11-25.

3 X. Ren, Y. Feng, J. Guo, et al., Surface modification and endothelialization of biomaterials as potential scaffolds for vascular tissue engineering applications, Chem. Soc. Rev., 2015, 44, 5680-5742.

4 T. M. Jeewandara, S. G. Wise and M. K. C. Ng, Biocompatibility of coronary stents, Materials, 2014, 7, 769-786.

5 S. P. Hoerstrup, G. Zünd, R. Sodian, et al., Tissue engineering of small caliber vascular grafts, Eur. J. Cardiothorac Surg., 2001, 20, 164-169.

6 E. T. Goh, E. Wong, Y. Farhatnia, et al., Accelerating in situ endothelialisation of cardiovascular bypass grafts, Int. J. Mol. Sci., 2014, 16, 597-627.

7 G. Wei, Q. Jin, W. V. Giannobile, et al., Nano-fibrous scaffold for controlled delivery of recombinant human PDGF-BB, $J$. Controlled Release, 2006, 112, 103-110.
8 S. Sahoo, L. T. Ang, J. C. H. Goh, et al., Growth factor delivery through electrospun nanofibers in scaffolds for tissue engineering applications, J. Biomed. Mater. Res., Part A, 2010, 93, 1539-1550.

9 W. Ji, Y. Sun, F. Yang, et al., Bioactive electrospun scaffolds delivering growth factors and genes for tissue engineering applications, Pharm. Res., 2011, 28, 1259-1272.

10 E. J. Carbone, T. Jiang, C. Nelson, et al., Small molecule delivery through nanofibrous scaffolds for musculoskeletal regenerative engineering, J. Nanomed. Nanotechnol., 2014, 10, 1691-1699.

11 C. Shi, F. Yao, Q. Li, et al., Regulation of the endothelialization by human vascular endothelial cells by ZNF580 gene complexed with biodegradable microparticles, Biomaterials, 2014, 35, 7133-7145.

12 C. Shi, F. Yao, J. Huang, et al., Proliferation and migration of human vascular endothelial cells mediated by ZNF580 gene complexed with mPEG- $b$-P (MMD-co-GA)- $g$-PEI microparticles, J. Mater. Chem. B, 2014, 2, 1825-1837.

13 J. Lv, J. Yang, X. Hao, et al., Biodegradable PEI modified complex micelles as gene carriers with tunable gene transfection efficiency for ECs, J. Mater. Chem. B, 2016, 4, 997-1008.

14 C. Shi, Q. Li, W. Zhang, et al., REDV peptide conjugated nanoparticles/pZNF580 complexes for actively targeting human vascular endothelial cells, ACS Appl. Mater. Interfaces, 2015, 7, 20389-20399.

15 Y. Feng, M. Guo, W. Liu, et al., Co-self-assembly of cationic microparticles to deliver pEGFP-ZNF580 for promoting the transfection and migration of endothelial cells, Int. J. Nanomed., 2017, 12, 137-149.

16 Q. Li, X. Hao, J. Lv, et al., Mixed micelles obtained by coassembling comb-like and grafting copolymers as gene carriers for efficient gene delivery and expression in endothelial cells, J. Mater. Chem. B, 2017, 5, 1673-1687.

17 H. Wang, Q. Li, J. Yang, et al., Comb-shaped polymer grafted with REDV peptide, PEG and PEI as targeting gene carrier for selective transfection of human endothelial cells, J. Mater. Chem. B, 2017, 5, 1408-1422.

18 J. Yang, X. Hao, Q. Li, et al., CAGW peptide-and PEGmodified gene carrier for selective gene delivery and promotion of angiogenesis in HUVECs in vivo, ACS Appl. Mater. Interfaces, 2017, 9, 4485-4497.

19 J. Yang, Q. Li, X. Yang, et al., Multitargeting gene delivery systems for enhancing the transfection of endothelial cells, Macromol. Rapid Commun., 2016, 37, 1926-1931.

20 H. Wang, Y. Feng, J. Yang, et al., Targeting REDV peptide functionalized polycationic gene carrier for enhancing the transfection and migration capability of human endothelial cells, J. Mater. Chem. B, 2015, 3, 3379-3391.

21 Q. Li, C. Shi, W. Zhang, et al., Nanoparticles complexed with gene vectors to promote proliferation of human vascular endothelial cells, Adv. Healthcare Mater., 2015, 4, 1225-1235.

22 X. Hao, Q. Li, J. Lv, et al., CREDVW-linked polymeric micelles as a targeting gene transfer vector for selective transfection and proliferation of endothelial cells, ACS Appl. Mater. Interfaces, 2015, 7, 12128-12140. 
23 L. De Laporte and L. D. Shea, Matrices and scaffolds for DNA delivery in tissue engineering, Adv. Drug Delivery Rev., 2007, 59, 292-307.

24 L. Peng, X. Cheng, R. Zhuo, et al., Novel gene-activated matrix with embedded chitosan/plasmid DNA nanoparticles encoding PDGF for periodontal tissue engineering, J. Biomed. Mater. Res., Part A, 2009, 90, 564-576.

$25 \mathrm{H}$. Storrie and D. J. Mooney, Sustained delivery of plasmid DNA from polymeric scaffolds for tissue engineering, $A d v$. Drug Delivery Rev., 2006, 58, 500-514.

26 L. Yu, Y. Feng, Q. Li, et al., PLGA/SF blend scaffolds modified with plasmid complexes for enhancing proliferation of endothelial cells, React. Funct. Polym., 2015, 91, 19-27.

27 Y. Feng, W. Liu, X. Ren, et al., Evaluation of electrospun PCLPIBMD meshes modified with plasmid complexes in vitro and in vivo, Polymers, 2016, 8, 58.

28 Y. K. Luu, K. Kim, B. S. Hsiao, et al., Development of a nanostructured DNA delivery scaffold via electrospinning of PLGA and PLA-PEG block copolymers, J. Controlled Release, 2003, 89, 341-353.

29 A. Hasan, A. Memic, N. Annabi, et al., Electrospun scaffolds for tissue engineering of vascular grafts, Acta Biomater., 2014, 10, 11-25.

$30 \mathrm{X}$. Hu, S. Liu, G. Zhou, et al., Electrospinning of polymeric nanofibers for drug delivery applications, J. Controlled Release, 2014, 185, 12-21.

31 S. Y. Chew, Y. Wen, Y. Dzenis, et al., The role of electrospinning in the emerging field of nanomedicine, Curr. Pharm. Des., 2006, 12, 4751-4770.

32 Q. P. Pham, U. Sharma and A. G. Mikos, Electrospinning of polymeric nanofibers for tissue engineering applications: a review, Tissue Eng., 2006, 12, 1197-1211.

33 J. Lannutti, D. Reneker, T. Ma, et al., Electrospinning for tissue engineering scaffolds, Mater. Sci. Eng., C, 2007, 27, 504-509.

34 H. Zhang, X. Jia, F. Han, et al., Dual-delivery of VEGF and PDGF by double-layered electrospun membranes for blood vessel regeneration, Biomaterials, 2013, 34, 2202-2212.

35 F. M. Chen, M. Zhang and Z. F. Wu, Toward delivery of multiple growth factors in tissue engineering, Biomaterials, 2010, 31, 6279-6308.

36 H. Cao, X. Jiang, C. Chai, et al., RNA interference by nanofiber-based siRNA delivery system, J. Controlled Release, 2010, 144, 203-212.

37 P. Rujitanaroj, Y. C. Wang, J. Wang, et al., Nanofibermediated controlled release of siRNA complexes for long term gene-silencing applications, Biomaterials, 2011, 32, 5915-5923.

38 M. Chen, S. Gao, M. Dong, et al., Chitosan/siRNA nanoparticles encapsulated in PLGA nanofibers for siRNA delivery, ACS Nano, 2012, 6, 4835-4844.

39 L. M. Bellan, E. A. Strychalski, H. G. Craighead, et al., Individually resolved DNA molecules stretched and embedded in electrospun polymer nanofibers, Nano Lett., 2006, 6, 2526-2530.

40 H. S. Kim and H. S. Yoo, MMPs-responsive release of DNA from electrospun nanofibrous matrix for local gene therapy: in vitro and in vivo evaluation, J. Controlled Release, 2010, 145, 264-271.

41 R. M. Raftery, E. G. Tierney, C. M. Curtin, et al., Development of a gene-activated scaffold platform for tissue engineering applications using chitosan-pDNA nanoparticles on collagenbased scaffolds, J. Controlled Release, 2015, 210, 84-94.

42 X. Zhao, D. E. Komatsu and M. Hadjiargyrou, Delivery of rhBMP-2 Plasmid DNA Complexes via a PLLA/Collagen Electrospun Scaffold Induces Ectopic Bone Formation, J. Biomed. Nanotechnol., 2016, 12, 1285-1296.

43 Y. Feng, M. Behl, S. Kelch, et al., Biodegradable multiblock copolymers based on oligodepsipeptides with shapememory properties, Macromol. Biosci., 2009, 9, 45-54.

44 W. Zhou, Y. Feng, J. Yang, et al., Electrospun scaffolds of silk fibroin and poly(lactide-co-glycolide) for endothelial cell growth, J. Mater. Sci.: Mater. Med., 2015, 26, 1-14.

45 J. Yang, M. Khan, L. Zhang, et al., Antimicrobial surfaces grafted random copolymers with REDV peptide beneficial for endothelialization, J. Mater. Chem. B, 2015, 3, 7682-7697.

46 C. Y. Wang, K. H. Zhang, C. Y. Fan, et al., Aligned naturalsynthetic polyblend nanofibers for peripheral nerve regeneration, Acta Biomater., 2011, 7, 634-643.

47 A. Barth, The infrared absorption of amino acid side chains, Prog. Biophys. Mol. Biol., 2000, 74, 141-173.

48 A. Kurane, D. T. Simionescu and N. R. Vyavahare, In vivo cellular repopulation of tubular elastin scaffolds mediated by basic fibroblast growth factor, Biomaterials, 2007, 28, 2830.

49 S. J. Hollister, Porous scaffold design for tissue engineering, Nat. Mater., 2005, 4, 518-524.

50 S. A. Wesolowski, C. C. Fries, K. E. Karlson, et al., Porosity: primary determinant of ultimate fate to synthetic vascular grafts, Surgery, 1961, 50, 91-96.

51 M. Zhu, K. Wang, J. Mei, et al., Fabrication of highly interconnected porous silk fibroin scaffolds for potential use as vascular grafts, Acta Biomater., 2014, 10, 2014-2023.

52 M. C. Phipps, W. C. Clem, J. M. Grunda, et al., Increasing the pore sizes of bone-mimetic electrospun scaffolds comprised of polycaprolactone, collagen I and hydroxyapatite to enhance cell infiltration, Biomaterials, 2012, 33, 524-534.

53 J. M. Anderson, A. Rodriguez and D. T. Chang, Foreign body reaction to biomaterials, Semin. Immunol., 2008, 20, 86-100.

54 M. M. Stevens and J. H. George, Exploring and engineering the cell surface interface, Science, 2005, 310, 1135-1138.

55 K. D. Andrews, J. A. Hunt and R. A. Black, Effects of sterilisation method on surface topography and in vitro cell behaviour of electrostatically spun scaffolds, Biomaterials, 2007, 28, 1014-1026.

56 J. M. Anderson, A. Rodriguez and D. T. Chang, Foreign body reaction to biomaterials, Semin. Immunol., 2008, 20, 86-100.

57 M. N. Avula, A. N. Rao, L. D. McGill, et al., Foreign body response to subcutaneous biomaterial implants in a mast cell-deficient $\mathrm{kit}^{\mathrm{w}-\mathrm{Sh}}$ murine model, Acta Biomater., 2014, 10, 1856-1863.

58 D. Ren, H. Wang, J. Liu, et al., ROS-induced ZNF580 expression: a key role for $\mathrm{H}_{2} \mathrm{O}_{2} / \mathrm{NF}-\kappa \mathrm{B}$ signaling pathway in vascular endothelial inflammation, Mol. Cell. Biochem., 2012, 359, 183-191. 